\title{
La generación y el potencial de transferibilidad del curriculum vitae en formato audiovisual
}

\begin{abstract}
Ana Landeta Etxeberria
Doctora en Dirección y Administración de Empresas (especialidad en estrategia corporativa y en estrategia y políticas de negocio) y directora del Instituto $I+D+i$ de la Universidad a Distancia de Madrid, UDIMA
\end{abstract}

ana.landeta@udima.es

Silvia Prieto Preboste

Coordinadora de la Unidad de Innovación de la Universidad a Distancia de Madrid, UDIMA

silvianazareth.prieto@udima.es

Este trabajo ha sido seleccionado para su publicación por: don Alfonso Aguiló Pastrana, doña Milagros Antón López, don Fernando Checa García, don Ángel de Miguel Casas, doña Laura Rayón Rumayor y don Javier Manuel Valle López.

\section{Extracto}

El curriculum vitae en formato audiovisual permite a cualquier demandante de empleo conocer estrategias y recursos personales adecuados para su inserción laboral y/o la mejora del puesto de trabajo, y, paralelamente, la adquisición de la competencia digital.

El presente trabajo se aborda desde dos perspectivas complementarias; como proceso, generación del curriculum vitae en formato audiovisual, y como resultado, transferencia directa del mismo a grupos objetivo de beneficiarios diferenciados: la sociedad, los estudiantes del ámbito de la formación profesional y de la formación superior, los grupos objetivo de ambos proyectos europeos de innovación educativa, el mundo empresarial y los refugiados que actualmente se encuentran en el campo de refugiados Nizip-2, en Turquía.

Así pues, lo fundamental y más relevante del resultado generado como producto final (videotutoriales para la generación de un curriculum vitae en formato audiovisual) es que la premisa de la transferencia del conocimiento se cumple, ya que el resultado-conocimiento originado tiene un potencial de transferibilidad notablemente alto.

Palabras clave: videocurrículum-branding; educación multimedia; competencias digitales; producción audiovisual; transferencia de conocimiento.

Cómo citar: Landeta Etxeberria, A. y Prieto Preboste, S. (2019). La generación y el potencial de transferibilidad del curriculum vitae en formato audiovisual. Tecnología, Ciencia y Educación, 13, 39-73. 


\title{
The generation and transferability potential of the curriculum vitae in audiovisual format
}

\author{
Ana Landeta Etxeberria \\ Silvia Prieto Preboste
}

\section{Abstract}

The curriculum vitae in audiovisual format allows any applicant to know suitable strategies and personal resources for their employability and/or their job position enhancement, considering the acquisition of digital competence.

This paper is addressed on two different perspectives; as a process, generating the curriculum vitae in audiovisual format, and as a result, transferring it directly to different beneficiaries: society, vocational and educational training students, higher education students, European innovative education projects, business sector and to refugees currently in the Nizip-2 refugee camp in Turkey.

Thus, the essential outcome (video tutorials for a curriculum vitae generation in audiovisual format) is that the knowledge transfer is achieved because the knowledge-outcome has generated a significant transferability potential.

Keywords: video curriculum-branding; multimedia education; digital skills; audiovisual production; knowledge transfer. 


\section{Sumario}

1. Introducción

2. Descripción de la generación del proceso y los resultados

3. Metodología

4. Nexo de resultados entre proyectos: generación de los resultados

4.1. Síntesis del desarrollo del proyecto CVTube

4.2. Síntesis del desarrollo del proyecto RefuSkills

5. Descripción del proceso de generación del curriculum vitae tutorial-audiovisual

5.1. Características generales de los resultados

5.2. Descripción del proceso

\subsubsection{Análisis}

5.2.2. Diseño del producto

5.2.3. Diseño instruccional

5.2.4. Diseño de producción

5.2.5. Preproducción

5.2.6. Desarrollo y producción

5.2.7. Integración, despliegue y testeo

6. Nexos de evaluación del resultado entre proyectos

7. Impacto de los resultados

7.1. Difusión de resultados asociados al proyecto CVTube

7.2. Difusión de resultados asociados al proyecto RefuSkills

8. La transferibilidad de los resultados y el conocimiento

9. Evaluación

10. Resultados

11. Conclusiones

Referencias bibliográficas 


\section{Introducción}

La innovación en los procesos de selección de personal pasa por el uso activo de uno de los métodos más novedosos que se encuentran las consultoras y los especialistas en recursos humanos a la hora de llevar a cabo dichos procesos: videocurrículum-branding (curriculum vitae en formato audiovisual).

Como principales ventajas de esta modalidad cabe destacar la brevedad, la concreción, la posibilidad de percibir más cosas que en una simple llamada telefónica y la adaptación de los procesos de selección a la evolución existente en el campo de las nuevas tecnologías.

El creciente uso de las redes online profesionales, así como la constante transformación digital que vive el mundo empresarial en la actualidad, presentan ventajas inherentes al acceso al mercado laboral. La existencia de herramientas y canales online más accesibles para los expertos en selección de personal y, al mismo tiempo, para el demandante de empleo propician el fomento del desarrollo de destrezas, de competencias digitales y de habilidades profesionales necesarias para que los candidatos demandantes de empleo dispongan de un acceso más rápido y efectivo a los potenciales reclutadores.

En este nuevo contexto laboral, una de las herramientas que ha adquirido más importancia a la hora de buscar empleo y, paralelamente, de generar una marca personal en internet, es el videocurrículum, y, sobre todo, el videocurrículum-branding o videocurrículum de marca personal, pues aporta un valor añadido incuestionable y logra la diferenciación del resto de los competidores. Como indica Climent-Rodríguez, Navarro-Abal y Ortega-Campos (2012), la realización de videocurrículums, es decir, vídeos en los que el demandante de empleo expone su currículum académico y profesional para optar a un puesto de trabajo, constituye una herramienta que puede facilitar la inserción laboral, ya que permite personalizar y singularizar al demandante de empleo frente al empleador. Esta nueva herramienta, además, posibilita que el demandante de empleo pueda mostrar otros factores cualitativos de su persona que, junto a sus datos curriculares, puedan suponer una ventaja a la hora de conseguir el puesto deseado.
En este nuevo contexto laboral, una de las herramientas que ha adquirido más importancia a la hora de buscar empleo es el videocurrículum

Este innovador formato, aparte de posicionar la marca personal de los sujetos en internet (por ejemplo, creación de un perfil en la red Linkedln), incrementa el nivel de oportunidades de acceso al mercado laboral $y$, por ende, mayores posibilidades de gestionar la carrera 
profesional; y, así mismo, permite a las empresas optimizar la captación del capital humano y los procesos de selección de personal, además de propiciar el fomento del desarrollo de competencias como futuros gestores de los recursos humanos y, por otro lado, sus posibilidades para realizar una adecuada visibilidad de la reputación profesional.

Los vídeos han sido considerados por muchos como una de las herramientas de instrucción en línea más efectivas. Según Treichler (1967), «la gente generalmente recuerda el $10 \%$ de lo que lee, el $20 \%$ de lo que escucha, el $30 \%$ de lo que ve y el $50 \%$ de lo que oye y ve». Muchos investigadores han investigado el papel del vídeo o del multimedia en el aprendizaje. La teoría de la codificación dual aborda las implicaciones del multimedia; de acuerdo con Paivio (1986) y Clark y Paivio (1991), la información se procesa a través de uno de dos canales: verbal o visual. El aprendizaje es mejor o más memorable si la información se procesa referencialmente a través de ambos canales. Los estudios de Mayer, Steinhoff, Bower y Mars (1995) y de Iding (2000) también llegaron a conclusiones similares.

En su mayor parte, hay dos tipos de instrucciones basadas en el vídeo: las realizadas con una cámara y las que se realizan con capturas de pantalla desde una computadora personal (pc). El primero, a menudo, se produce en un ambiente tipo aula donde un instructor da una conferencia y el público escucha. La mayoría de los vídeos instructivos pertenecen a esta categoría. Esto último implica el uso de un software de captura de pantalla. Un vídeo de demostración de captura de pantalla típico muestra movimientos pregrabados en pantalla, que a menudo se sincronizan con las narraciones del instructor. Los vídeos de captura de pantalla han ganado popularidad gracias a internet. A medida que la web se está convirtiendo en el medio preferido de aprendizaje, los contenidos objetivo y el entorno de aprendizaje (por ejemplo, la interfaz, las navegaciones) se entrelazan cada vez más. Por lo tanto, no sorprende que cada vez más capacitadores y educadores estén utilizando aplicaciones de captura de pantalla para crear sus tutoriales en línea.

Se puede usar una teoría de carga cognitiva para explicar el éxito de las soluciones de vídeo de captura de pantalla o herramientas similares. Según Sweller y Chandler (1994), cuando los estudiantes se ven obligados a integrar mentalmente la información de fuentes de información separadas, su carga cognitiva se incrementa innecesariamente (conocido como el «efecto de atención dividida»). Un buen ejemplo de esto es la frustración de tratar de referirse de un lado a otro entre el texto colocado por separado y el diagrama que lo acompaña; la separación entre los dos dificulta la comprensión. Como indica el estudio de Sweller y Chandler, para que los estudiantes aprendan mejor, sería útil proporcionar los objetos representados lo más cerca posible de la descripción. En el contexto de soporte bajo demanda, un buen soporte proporciona al solicitante ayuda relevante y contextual de manera oportuna.

Algunos estudios empíricos revelan la importancia que posee el modelo de producción de vídeo en la capacidad de engagement que tiene en los estudiantes. Algunos de los rasgos que hacen más efectivos los vídeos son la menor duración, cierta informalidad, alternar diapositivas con la imagen del profesor y los discursos rápidos y entusiastas (Guo, Kim y Rubin, 2014). 


\section{Descripción de la generación del proceso y los resultados}

Contexto del proceso asociado a la generación de los resultados (generación del curriculum vitae en formato audiovisual y, como resultado, transferencia directa del mismo a grupos objetivo de beneficiarios diferenciados): dos proyectos europeos de innovación educativa dentro del marco del Programa Erasmus $+{ }^{1}$ que se describen someramente a continuación.

Cuadro 1. Descripción general de los proyectos europeos de innovación educativa CVTube y RefuSkills

\section{Proyecto CVTube}

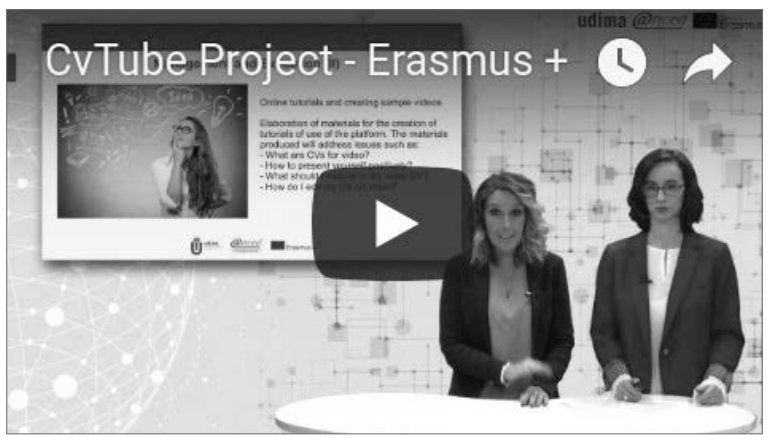

Descripción general del proyecto disponible en el canal YouTube de la UDIMA:

$<$ https://www.youtube.com/watch?v=7e1UDHjlngs.

Convocatoria: KA2-Cooperation for Innovation and the Exchange of Good Practices Strategic Partnerships for Vocational Education and Training.

Número de referencia (expediente): 2016-1-CZ01-KA202-024024.

El proyecto CVTube consiste en la creación de una plataforma online a través de la cual los alumnos de formación profesional podrán presentar sus curriculum vitae en formato vídeo.

1 El Programa Europeo de Educación, Formación, Juventud y Deporte 2014-2020 Erasmus+ es el programa europeo en los ámbitos de la educación, la formación, la juventud y el deporte para el periodo 20142020. Erasmus+ es el programa que trata de impulsar las perspectivas laborales y el desarrollo personal, además de ayudar a nuestros sistemas de educación, formación y juventud a proporcionar una enseñanza y un aprendizaje que doten a las personas de las capacidades necesarias para el mercado laboral y la sociedad actual y futura. 


\section{Proyecto CVTube}

Los objetivos de este proyecto son, entre otros:

- Mejorar la empleabilidad de los alumnos.

- Ayudarles a aprender nuevas habilidades (edición de vídeo, técnicas para la búsqueda de empleo, trucos para la realización de un buen curriculum vitae, etc.).

- Enseñarles a enfrentarse a una entrevista de trabajo.

La UDIMA participa en este proyecto como colaboradora con la Asociación Nacional de Centros de E-Learning y Distancia (ANCED) (<https://www.anced.es/>), que es socia, junto a estas otras entidades:

- EuroProfis (coordinador, República Checa): <http://www.europrofis.cz/>.

- EDIT VALUE (Portugal): <http://www.editvalue.com/>.

- WH Gelsenkirchen (Alemania): <https://www.w-hs.de/>.

- Obchodni Akademie (República Checa): <http://oa-pisek.cz/>.

- Cámara de Comercio de Badajoz (España): <http://www.camarabadajoz.es/web/>.

- Universidade do Minho (Portugal): <https://www.uminho.pt/PT>.

Metas:

- Desarrollar una plataforma que pueda ayudar a aumentar la empleabilidad de los jóvenes.

- Acercar al sector empresarial y los posibles empleadores.

- Aumentar la movilidad de aquellos jóvenes que no puedan encontrar trabajo en su país de origen.

Plataforma CVTube (principal resultado del proyecto):

- Plataforma amigable para los perfiles profesionales de jóvenes y videocurrículums.

- Una herramienta interactiva (compatible con smartphones y tablets).

- Conexión (entre solicitantes de empleo y el mercado de trabajo).

Beneficiarios:

- Jóvenes de la Unión Europea demandantes de empleo (primer empleo o mejora del actual).

- Buscadores de empleo (ciudadanos en búsqueda activa de empleo).

- Departamentos de recursos humanos (reclutadores, expertos en selección de personal, etc.).

- Profesorado del ámbito de la formación profesional y de la formación superior.

- Servicios Nacionales de Empleo.

- Agencias nacionales de reclutamiento.

Web del proyecto CVTube: <http://project.cvtuber.eu/es/>. 


\section{Proyecto RefuSkills}

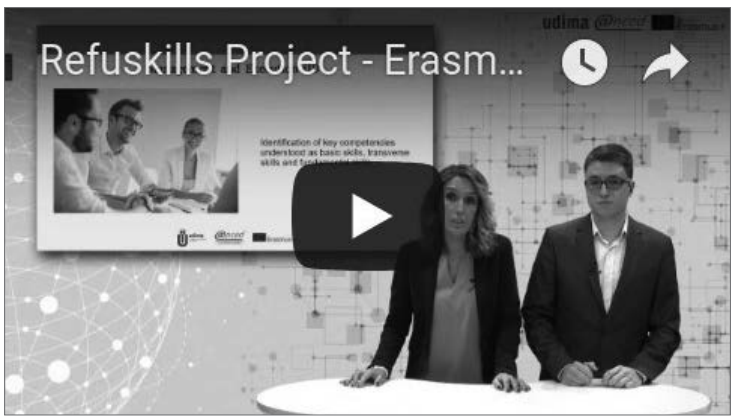

Descripción general del proyecto disponible en el canal YouTube de la UDIMA:

$<$ https://www.youtube.com/watch?time_continue=24\&v=AApVj-vJ6No $>$.

Convocatoria: KA2-Cooperation for Innovation and the Exchange of Good Practices Strategic Partnerships for Vocational Education and Training.

Número de referencia (expediente): 2016/1/NO01/KA202/022083.

El proyecto RefuSkills consiste en la adaptación de una plataforma de reconocimiento y validación de competencias académicas y profesionales adaptadas a las necesidades de los refugiados "sin papeles" que viven en los campos de refugiados en Europa en el momento presente. La plataforma resultante permitirá a los mismos ver reconocidas sus habilidades y competencias con el fin de mejorar su empleabilidad y movilidad laboral dentro y fuera del espacio de la Unión Europea.

\section{Objetivos:}

- Establecer un mecanismo fácil de utilización que dé a los refugiados la oportunidad de documentar sus habilidades y competencias (en su propio idioma).

- Facilitar y reducir el tiempo de procesamiento en la solicitud de permisos de asilo, e identificar el camino adecuado hacia la formación y el empleo.

- Mejorar las posibilidades de los países receptores para una rápida identificación de las habilidades y de las competencias de los migrantes, así como facilitar su integración y participación en programas de creación de trabajo.

- Explorar la posibilidad de utilizar un "RefuPass» que establezca un porfolio de competencias individuales basadas en resultados de aprendizaje.

La UDIMA participa en este proyecto como colaboradora con la ANCED (<https://www.anced.es/>), que es socia, junto a estas otras entidades:

- NTI-MMM (coordinador, Noruega): <https://www.ntim.eu/>.

- ISQ (Italia): <https://www.isq.pt/ES/>. 


\section{Proyecto RefuSkills}

- EureCons (Alemania): <https://www.eurecons.com/index.php/en/>.

- KALIBAO (Francia): <http://www.kalibao.com/>.

- WIAB (Austria): <http://www.wiab.at/index.html>.

- BW (Austria): <https://www.bfi.wien/>.

- Associazione Comunità Papa Giovanni XXIII (Italia): <https://www.apg23.org/>.

- EUROMASC (Noruega): <https://euromasc.org/>.

- SBG Dresden (Alemania): <https://www.sbg-dresden.de/>.

- EWF (Bélgica): <https://www.ewf.be/>.

- Istanbul II Sosya (Turquía): <http://istanbulproje.gov.tr/>.

- HIOA (Noruega): <http://www.hioa.no/>.

- AW (Austria): <https://www.ams.at/>.

Objetivos específicos del proyecto:

- Establecimiento de un mecanismo fácil de usar que brinde a los refugiados/emigrantes la oportunidad de documentar sus habilidades y competencias (en su propio idioma) mediante el uso de los principios básicos del sistema europeo de créditos para la educación y la formación profesionales (ECVET) y de los resultados de aprendizaje.

- Facilitación y reducción del tiempo de tramitación en el manejo de las solicitudes de residencia de los refugiados y asilados, e identificación de trayectorias individuales de carrera más adecuadas para el empleo y la capacitación.

- Mejorar las posibilidades de los países receptores para realizar una rápida identificación de las habilidades y de las competencias de los emigrantes y, por lo tanto, mejorar la integración y la participación en los programas de trabajo y desarrollo de capacidades.

- Explorar la posibilidad de emitir un RefuPass que establezca la cartera de competencias individuales basada en el aprendizaje.

Beneficiarios:

- Los campamentos de refugiados y los centros de acogida.

- Los centros de compensación para refugiados y solicitantes de asilo que preparan su integración.

- Los servicios de empleo y las oficinas de la Seguridad Social.

- Las organizaciones/organizaciones no gubernamentales (ONG) humanitarias activas en este campo.

- Las organizaciones de capacitación, las escuelas y los centros de formación profesional a nivel regional y nacional.

- Los lugares de trabajo que recluten refugiados y solicitantes de asilo.

- Autoridades nacionales responsables de la migración, la integración y el empleo de los países del consorcio.

- Organizaciones y autoridades transnacionales y europeas, como la Dirección General de Empleo, Asuntos Sociales e Inclusión y el Departamento de Ayuda Humanitaria y Protección Civil de la Comisión 


\section{Proyecto RefuSkills}

Europea (ECHO). La United Nations Educational, Scientific and Cultural Organization (UNESCO) y la United Nations International Children's Emergency Fund (UNICEF) también serán debidamente informadas e invitadas a considerar a RefuSkills como un instrumento para la inclusión social y la integración de primera magnitud.

Web del proyecto RefuSkills: <https://skillstools.eu/refuskills/>. Información disponible en la web de la UDIMA/Sección Investigación/Innovación/Proyectos: <https://www.udima.es/es/innovacion/proyectos. $h t m l>$.

Fuente: elaboración propia a partir de la memoria técnica del proyecto.

\section{Metodología}

El diseño metodológico se fundamenta en una aplicación adaptada de los procesos de investigación-acción (IA) (Rodríguez y González, 2013) para lograr la mejora de los programas educativos y, de forma especial, los sistemas de planificación y desarrollo de esta tipología de proyectos que contienen numerosos recursos educativos en soporte multimedia (videotutoriales derivados de la producción audiovisual intrínseca al propio diseño instruccional). En un contexto de fuerte cambio en las dinámicas educativas a nivel global (González-Sancho y Vincent-Lancrin, 2016), sometidas a una adaptación de los procesos de aprendizaje a las lógicas líquidas de una sociedad globalizada (Best, 2015), resulta esencial realizar aproximaciones críticas que, a partir de una interacción constante y retroalimentada entre conocimiento y actuación, permitan obtener los mejores resultados en términos prácticos, adaptándose estos a las necesidades reales tanto del contexto en el que se desarrollan como a la comunidad de destino.

Siguiendo la secuencia de las fases y la evaluación estándar de la IA definidas por Lewin (citado en Elliot, 2005), desde una perspectiva de aplicación concreta al desarrollo de productos similar a la realizada por otras investigaciones (Contreras, Eguia y Solano, 2016), se presenta el modelo metodológico alcanzado en la producción y en el desarrollo de contenidos audiovisuales y multimedia para generar videotutoriales que permitan el reconocimiento de las habilidades profesionales y académicas de los estudiantes, así como la adquisición de las competencias digitales, a través de la generación de interacciones sucesivas basadas en ciclos recursivos de planificación, acción, observación y reflexión.

Se ha optado por este enfoque metodológico porque permite abrir la participación a todos los equipos involucrados en el proceso de elaboración de los videotutoriales, tiene una clara orientación práctica que facilita aprender del proceso y de los errores cometidos durante este, y permite evolucionar en espiral en la medida en que crece la 
dimensión del proyecto y su alcance (Given, 2008). El principal objetivo ha radicado en generar unos recursos educativos reusables y transferibles al ámbito educativo y empresarial propiciando el acceso al mercado laboral del usuario final. De este modo, el proceso se ha llevado a cabo durante el desarrollo secuencial descrito en detalle en el apartado 5 (Descripción del proceso de generación del curriculum vitae tutorial-audiovisual) del presente trabajo, dentro de la estrategia general de desarrollo del proyecto CVTube, transfiriendo el resultado núm. 2/videotutoriales al resultado núm. 6 del proyecto RefuSkills.

El desarrollo de un curriculum vitae en formato audiovisual requiere de un proceso de planificación, diseño y desarrollo que implica a diferentes profesionales y áreas de trabajo. La eficacia del sistema de producción precisa establecer metodologías específicas que atiendan no solo a las características concretas del contexto de elaboración, sino que aúnen estrategias y técnicas procedentes de diferentes ámbitos, como el diseño de recursos educativos, la producción audiovisual y el desarrollo multimedia e interactivo. A través de la aplicación de un proceso inspirado en los principios de investigación-acción, se presenta un modelo metodológico para la producción y el desarrollo de contenidos audiovisuales y multimedia. Un proceso que se ha llevado a cabo durante el desarrollo de la generación de resultados asociados al proyecto europeo Erasmus+: CVTube. El proceso ha requerido la configuración de equipos de trabajo y medios especializados, de un programa de acción y de la definición de estrategias que han sido puestas a prueba mediante procesos iterativos y de diseño, implantación (acción), análisis y evaluación, dirigidos a la mejora y a la eficacia de los procesos de producción. La secuencia de interacciones ha permitido definir un modelo de producción más ligero, basado en el desarrollo de esquemas y patrones formales que funcionan como plantillas generales tanto para la producción audiovisual como multimedia, y la mejora de los flujos de comunicación y trabajo entre los equipos involucrados. Una metodología afín a la producción para el desarrollo de contenidos audiovisuales y multimedia para los massive open online course (MOOC) propuesta por Gértrudix, Rajas y Álvarez (2017).

Al respecto, Paulson, Paulson y Meyer (1991) señalan que, cuando lo confecciona el alumnado, debe incluir su participación en la selección del contenido, los criterios de selección, el criterio para evaluar los méritos y la evidencia de la autorreflexión llevada a cabo.

Así, la apuesta por un modelo de producción determinado se establece a partir de tres vertientes:

- Tecnológica. Valora y selecciona los medios técnicos digitales más apropiados para la creación y distribución de contenidos.

- Narrativa y estética. Toma decisiones en el ámbito de la construcción de relatos audiovisuales originales, desde la escritura del guion a la posproducción, pasando por la dirección de actores, la fotografía o el diseño sonoro.

- Didáctica. Integra y guía la producción de materiales en función de su finalidad específicamente formativa. 


\section{Nexo de resultados entre proyectos: generación de los resultados}

En síntesis, en el presente apartado se describen, por un lado, las fases de desarrollo de los proyectos $\mathrm{y}$, finalmente, el nexo (resultado final: videotutoriales para la generación de videocurrículums) de transferencia de los resultados entre ambos proyectos, así como la transferencia del conocimiento-resultado a los diferentes beneficiarios de los mismos a través de los planes de difusión de resultados previstos para ambos proyectos.

\subsection{Síntesis del desarrollo del proyecto CVTube \\ El proyecto CVTube se ha llevado a cabo en cuatro fases:}

\section{Cuadro 2. Fases del proyecto CVTube}

\section{Fase I. Análisis del mercado laboral y requisitos técnicos}

Tareas y objetivos específicos asociados

01/T1. Revisión del estado de la cuestión y análisis del mercado laboral:

- 1 informe que especifica los grupos destinatarios, la comprensión del mercado laboral, los servicios a medida y la perspectiva del aprendizaje permanente.

- 20 entrevistas de expertos con empleadores.

01/T2. Definición de los requisitos y conclusiones:

- 1 informe sobre los requisitos para la plataforma CVTube desde la perspectiva de los empleadores, ejemplos de buenas prácticas y valor educativo añadido.

Fase II. Tutoriales en línea y programas inteligentes para la creación de vídeos (open educational resources [OER])

Tareas y objetivos específicos asociados

02/T1. Definición de estándares de calidad:

- 1 catálogo con estándares para la realización técnica, el diseño, la usabilidad, etc.

02/T2. Seminarios de investigación dirigidos a estudiantes:

- 2 seminarios dirigidos a estudiantes de formación profesional en checo y español.

- 2 seminarios dirigidos a estudiantes de formación superior en alemán y portugués.

- 4 resúmenes de requisitos técnicos. 
(cont.) Fase II.

02/T3-T5. Diseño y creación de tutoriales y vídeos de ejemplo:

- 1 guion y 1 diseño conceptual para tutoriales.

- 30 vídeos de ejemplo.

- Mínimo 5 tutoriales grabados.

- 1 manual escrito con toda la información de los tutoriales.

02/T6. Evaluación interna y finalización de los tutoriales online:

- 1 informe de evaluación de cada socio (7 en total).

- Tutoriales mejorados en los idiomas inglés, alemán, checo, español y portugués.

Fase III. CVTube plataforma online para curriculum vitae en vídeo

Tareas y objetivos específicos asociados

03/T1. Desarrollo del software y la plataforma:

- Prototipo de plataforma.

03/T2-T3. Pruebas, ajustes y finalización de la prueba piloto de la plataforma online:

- 2 pruebas piloto (mínimo 40 participantes de formación profesional/20 participantes universitarios).

- 2 informes de retroalimentación por cada prueba.

- Plataforma finalizada.

Fase IV. Manual de uso y capacitación + Materiales adicionales para contactar exitosamente con los empleadores

Tareas y objetivos específicos asociados

04/T1. Elaboración del diseño instruccional y manual de capacitación:

- 1 manual para profesores, consultores, servicios de empleo y otros stakeholders.

04/T2. Pruebas piloto del manual de uso y capacitación:

- 4 pruebas piloto (1 en cada país del consorcio).

- Una lista de 20 ejemplos y mejores prácticas para convencer a los empleadores con personalidad.

- 20 ejemplos, sugerencias y mejores prácticas para mejorar habilidades interpersonales y cómo mostrarlas en un perfil en vídeo.

- 20 ejercicios creativos para ayudar a los jóvenes a perder el miedo frente a una cámara.

04/T3. Finalización del manual de uso y capacitación:

- Instrucciones técnicas sobre cómo usar la plataforma CVTube y editar los vídeos.

- Orientación competente para los profesionales.

Fuente: elaboración propia a partir de la memoria técnica del proyecto. 


\subsection{Síntesis del desarrollo del proyecto RefuSkills}

Por su parte, el proyecto RefuSkills se llevó a cabo en las siguientes fases:

\section{Cuadro 3. Fases del proyecto RefuSkills}

Fase I. Descripción general de la situación de los refugiados y ejemplos de buenas prácticas de políticas para identificar, registrar y evaluar las competencias de los refugiados

Tareas y objetivos específicos asociados

01/T1. Información general sobre la situación de los refugiados en los países socios (Austria, Alemania, España, Francia, Italia, Noruega, Portugal y Turquía):

Recopilación de información general sobre la situación de los refugiados en los países socios (Austria, Alemania, España, Francia, Italia, Noruega, Portugal y Turquía). Por ejemplo: datos básicos sobre el número de refugiados que llegaron al país, de qué países provienen, etc.

01/T2. Recopilación de buenas prácticas, medidas y políticas para registrar y evaluar las competencias de los refugiados en los países socios seleccionados:

- Centrándose en los países socios que acogieron a más refugiados desde el verano de 2015: Austria, Alemania y Noruega. Pero también teniendo en cuenta los ejemplos de buenas prácticas que se han recopilado durante la primera tarea en otros países socios (España, Italia, Portugal y Turquía).

\section{Fase II. Identificación de las competencias clave}

\section{Tareas y objetivos específicos asociados}

02/T1. Visión general de los marcos existentes en los países socios, agregando información de otras fuentes, como las definiciones de las competencias clave de la Unión Europea y la estructuración de la Organización para la Cooperación y el Desarrollo Económicos (OCDE) para las evaluaciones del Programme for the International Assessment of Adult Competencies (PIAAC). Por último, se trabajarán los diferentes marcos para que estén orientados a resultados de aprendizaje y permitan una alineación más fácil con los principios del ECVET.

02/T2. Basado en la primera tarea, se prepara un estudio comparativo de los distintos enfoques. Esta comparación debe conducir a la elaboración de un borrador de la matriz que abarque los diferentes marcos. En la medida de lo posible, la matriz debería organizarse en unidades lógicas de resultados de aprendizaje.

02/T3. La matriz propuesta se distribuye entre los socios para su consulta y se presenta en común en una de las reuniones del proyecto, validando la propuesta matriz modificada en aras a la construcción de la versión final. 
(cont.) Fase II.

02/T4. Tareas desarrolladas en tres fases diferenciadas:

- Traducción de la versión en inglés a todos los idiomas de los socios del proyecto.

- Traducción del inglés al árabe.

- Publicación de todas las versiones al Skillsbank (compilador de cursos y competencias profesionales noruego asociado al ECVET).

02/T5. Entrega de un informe.

Fase III. Identificación de las ocupaciones más demandadas o relevantes para los refugiados

Tareas y objetivos específicos asociados

03/T1. Establecimiento de una visión general de las ocupaciones más frecuentes de los refugiados en los países socios. Cada socio "nomina» al menos 10 ocupaciones diferentes y la descripción de su calificación. Un criterio adicional para la nominación será que los resultados de aprendizaje tengan algunas similitudes al formato ECVET, ya que esto será necesario para los siguientes pasos. La descripción de cada ocupación en formato "certificado de profesionalidad» se realiza en todo los idiomas de los países socios.

03/T2. Calificación de las ocupaciones nominadas (profesiones del tipo camarero, peluquero, administrativo, etc.) de acuerdo con el ECVET, organizadas por grupos temáticos profesionales y competenciales.

03/T3. Presentación del borrador de la colección de ocupaciones-profesionales y marcos competenciales de acuerdo a la naturaleza de la matriz definida previamente a través de un informe a todos los socios para recabar sus comentarios y aportaciones y trasladarlo a la siguiente fase.

\section{Fase IV. Selección de una matriz de calificación basada en las descripciones} de calificación de los países participantes

Tareas y objetivos específicos asociados

04/T1. Basándose en el material recopilado de los socios, las calificaciones nominadas se evalúan según Ios criterios ECVET y se selecciona un núcleo de calificaciones para su posterior desarrollo y traducción. Para el propósito del proyecto, la selección de calificaciones/ocupaciones debe tener en cuenta el sesgo de género implícito, asegurando que las ocupaciones «típicas» de hombres y mujeres no dominen.

04/T2. Las calificaciones seleccionadas se deben simplificar de acuerdo con los resultados de aprendizaje y se deben preparar para su traducción y publicación en el Skillsbank. 
(cont.) Fase IV.

04/T3. Desarrollo de tareas a tres niveles:

- Traducción de la versión en inglés a todos los idiomas de los socios del proyecto.

- Traducción del inglés al árabe.

- Publicación de todas las versiones al Skillsbank.

04/T4. Entrega del informe.

Fase V. Reconocimiento del aprendizaje previo, abarcando el aprendizaje formal, no formal e informal adquirido en diferentes entornos

Tareas y objetivos específicos asociados

05/T1. Revisión de los procedimientos de reconocimiento del aprendizaje previo que actualmente están funcionando en los entornos de refugiados y, especialmente, aquellos basados en los principios ECVET.

05/T2. Desarrollar/refinar los procedimientos y principios del Skillsbank para evaluar y documentar el aprendizaje previo en lo que respecta a las habilidades básicas y a las competencias clave.

05/T3. Desarrollar/refinar los procedimientos y principios de Skillsbank para evaluar y documentar el aprendizaje previo en lo que respecta a las calificaciones de las ocupaciones.

05/T4. Desarrollo de directrices y un handbook para la implementación de todos los resultados generados.

\section{Fase Vl. RefuSkills folletos, asociados al «Pasaporte de Competencias/RefuPass»}

\section{Tareas y objetivos específicos asociados}

06/T1. Definición del contenido y formato del «Pasaporte de Competencias/RefuPass».

06/T2. Definición del contenido y formato de los posibles documentos y certificados relacionados con el curriculum vitae Europass.

06/T3. Definición del contenido y del formato de los posibles archivos y vídeos para documentar las habilidades y competencias.

06/T4. Revisión y modificación de la documentación tras las pruebas piloto realizadas en los diferentes países del consorcio. 
(cont.) Fase VI.

Fase VII. Especificaciones de interacción del sistema para optimizar el uso del Skillsbank en un entorno en el que el usuario final realiza una autoevaluación encaminada a un reconocimiento del aprendizaje previo

\section{Tareas y objetivos específicos asociados}

07/T1. Revisión de la documentación generada en la primera fase.

07/T2. Revisión de la documentación generada en las fases 5 y 6 y definición de contenido y estructura para un módulo de recuperación de estadísticas.

07/T3. Exploración de la posibilidad de integrar dispositivos móviles para la interacción entre el usuario final y el sistema Skillsbank.

07/T4. Preparación de maquetas para el equipo de desarrollo.

\section{Fase VIII. Desarrollo del sistema}

Estructura de datos que asegure que todos los aspectos relevantes de entrada, proceso, almacenamiento y salida se ajustan a los objetivos principales de RefuSkills

08/T1. Revisión de la información de la fase anterior para realizar las modificaciones necesarias y la integración con las funciones existentes de Skillsbank/Skillstube.

08/T2. Definición de la estructura de datos.

08/T3. Definición de las opciones de API-WEB (para dispositivos móviles).

08/T4. Testeo de diferentes opciones para un diseño web adaptativo.

08/T5. Preparar el plan de desarrollo y producción.

Fase IX. Desarrollo del sistema: modificaciones de la interfaz y adaptaciones

Tareas y objetivos específicos asociados

09/T1. Desarrollo de las modificaciones necesarias según el resultado obtenido en las conclusiones de la anterior fase.

09/T2. Desarrollo de soluciones para dispositivos móviles.

09/T3. Desarrollo de una interfaz de acceso. 
(cont.) Fase IX.

09/T4. Desarrollo de las modificaciones necesarias en un entorno operativo de derecha a izquierda (escritura árabe).

09/T5. Finalización del desarrollo con la retroalimentación recibida en la fase anterior.

Fase X. Testeo de implementación en los países socios

\section{Tareas y objetivos específicos asociados}

010. ResfuSkills. Desarrollo de un testeo por pasos en los países socios para depurar errores, hacer modificaciones y mejorar la funcionalidad.

010/T1. Preparar los procedimientos de testeo en los países socios basándose en la información de 01, O2, 04 y 09.

010/T2. Revisión de los procedimientos de testeo.

010/T3. Recopilación de los comentarios de retroalimentación.

010/T4. Dar la retroalimentación sobre las modificaciones propuestas y la depuración de errores al equipo de desarrollo.

Fase XI. Implantación A: en los países asociados y otros organismos relevantes e interesados a nivel nacional

Tareas y objetivos específicos asociados

011/T1. Recopilación de la información de las anteriores fases para definir los resultados e instrucciones operativas de la metodología aplicada. Preparación de un manual (handbook) para la implementación.

011/T2. Motivación, seguimiento y apoyo a las organizaciones implementadoras.

011/T3. Dar retroalimentación a los socios del proyecto sobre el progreso y las modificaciones propuestas. Preparar el informe de implementación A.

Fase XII. Implementación B: en organizaciones externas a los países asociados

Tareas y objetivos específicos asociados

012/T1. Recopilación de la información de las anteriores fases para definir los resultados e instrucciones operativas de la metodología aplicada. Preparación de un manual para la implementación. 
(cont.) Fase XII.

012/T2. Consulta con los socios sobre la selección del grupo objetivo, en función de su red de contactos para llegar a posibles organizaciones de implementación.

012/T3. Motivación, seguimiento y apoyo a las organizaciones implementadoras.

012/T4. Facilitar retroalimentación a los socios del proyecto sobre el progreso y las modificaciones propuestas. Preparar el informe de implementación A.

Fuente: elaboración propia a partir de la memoria técnica del proyecto.

Cuadro 4. Proceso de producción de videotutoriales asociados a la fase II del programa CVTube e incluidos en el formulario vinculado a la conformación del «Pasaporte de Competencias/ RefuPass» (06/T4)

\section{Características generales de los resultados}

02/T1. Definición de estándares de calidad:

- Resultado I. 1 catálogo con estándares para la realización técnica, el diseño, la usabilidad, etc.

02/T2. Seminarios de investigación dirigidos a estudiantes:

- Resultado II. 2 seminarios dirigidos a estudiantes de formación profesional en checo y español.

- Resultado III. 2 seminarios dirigidos a estudiantes de formación superior en alemán y portugués.

- Resultado IV. 4 resúmenes de requisitos técnicos.

02/T3-T5. Diseño y creación de tutoriales y vídeos de ejemplo:

- Resultado V. 1 guion y 1 diseño conceptual para tutoriales.

- Resultado VI. 30 vídeos de ejemplo.

- Resultado VII. Mínimo 5 tutoriales grabados.

- Resultado VIII. 1 manual escrito con toda la información de los tutoriales.

02/T6. Evaluación interna y finalización de los tutoriales online:

- Resultado IX. 1 informe de evaluación de cada socio (7 en total).

- Resultado X. Tutoriales mejorados en inglés, alemán, checo, español y portugués.

06/T4. Revisión y modificación de la documentación tras las pruebas piloto realizadas en los diferentes países del consorcio. 


\section{Descripción del proceso de generación del curriculum vitae tutorial-audiovisual}

\subsection{Características generales de los resultados}

Los videotutoriales constituyen el resultado núm. 2 del proyecto CVTube. Dirigido por este diseño de resultados generales asociados al proceso de generación del curriculum vitae tutorial-audiovisual, se organiza en un ciclo que se sintetiza en la siguiente figura:

Figura 1. Proceso de generación de los videotutoriales

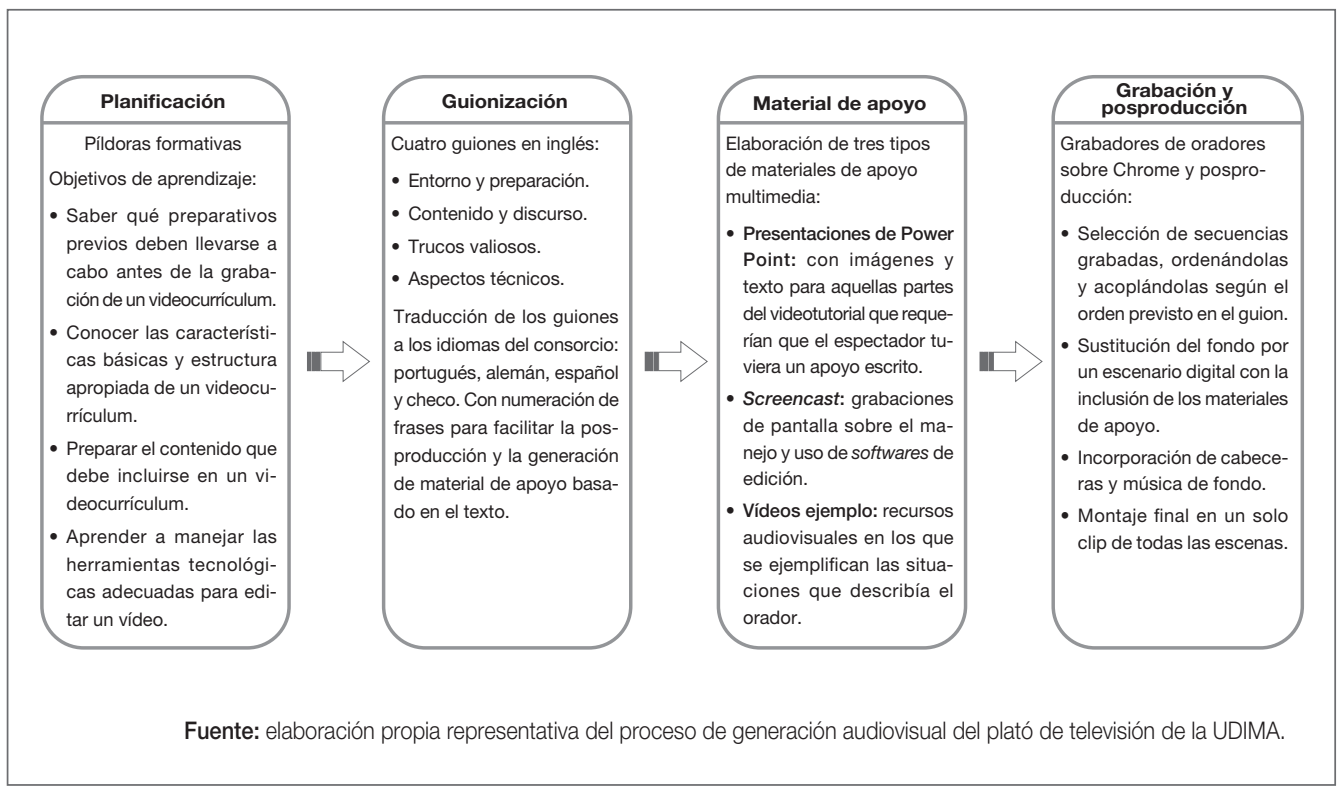

Se trata de un proceso en constante evolución, basado en un modelo metodológico híbrido de desarrollo, que es fruto del contexto de producción en el que se desenvuelve.

\subsection{Descripción del proceso}

\subsubsection{Análisis}

Las tareas de análisis constituyen la pieza clave del desarrollo del curriculum vitae tutorialaudiovisual. Si bien, desde una perspectiva teórica, estas tareas de análisis podrían con- 
siderarse un punto de partida, en la práctica, la realidad es que están presentes en todos los momentos del diseño, de la producción y del seguimiento.

\subsubsection{Diseño del producto}

Este tipo de vídeo permite una mejor asimilación de los conceptos tratados (Mayer y Moreno, 2007) y se caracteriza por ser de duración limitada (1-5 min), por incluir contenidos multimedia (Borrás, 2012) y por tener un propósito educativo muy concreto y unos objetivos de aprendizaje muy definidos.

\subsubsection{Diseño instruccional}

Realizado por docentes expertos en el área de conocimiento asociada a los procesos de selección de las áreas de dirección y gestión de recursos humanos. Los objetivos de aprendizaje establecidos fueron:

- Saber qué preparativos previos deben llevarse a cabo antes de la grabación de un videocurrículum.

- Conocer las características básicas y la estructura apropiada de un videocurrículum.

- Preparar el contenido que debe incluirse en un videocurrículum.

- Aprender a manejar las herramientas tecnológicas adecuadas para editar un vídeo.

\subsubsection{Diseño de producción}

Estrechamente ligado al diseño de producto, comparte su naturaleza dinámica y flexible. La planificación detallada y la normalización de procesos conviven de forma óptima con la flexibilidad necesaria para la prevención de problemas y desajustes en costes y en uso de recursos.

\subsubsection{Preproducción}

Esta es una de las fases que mayor evolución ha experimentado desde el trabajo en cascada inicial a los actuales métodos, que implican la colaboración entre los equipos docentes y los equipos de producción audiovisual y multimedia. Se consigue, de esta manera, una mayor agilidad en el desglose de los contenidos y los guiones audiovisuales y multimedia. 
La planificación compleja y detallada deja paso a la retroalimentación directa en reuniones de trabajo en las que las necesidades de producción se unen con los primeros pasos en el desarrollo de contenidos audiovisuales y multimedia. En función de los objetivos de aprendizaje establecidos en el diseño instructivo se elaboraron guiones para cuatro videotutoriales:

- Entorno y preparación. Contiene instrucciones sobre los materiales necesarios para llevar a cabo la grabación, el lugar óptimo para hacerlo, la iluminación y las recomendaciones sobre cómo deben prepararse este tipo de vídeos.

- Contenido y discurso. En este vídeo se explican detalles del contenido de un videocurrículum: duración, estructura, formación que se debe incluir, aspectos de la experiencia profesional o de las habilidades personales que hay que destacar, etc.

- Trucos valiosos. Consejos sobre cómo realizar la grabación: normas de etiqueta, vocabulario adecuado, actitud ante la cámara o tono del discurso, etc.

- Aspectos técnicos. Describe aquellas herramientas y técnicas necesarias para llevar a cabo la edición de los videocurrículums.

En la redacción de los guiones se llevaron a cabo las acciones establecidas por Borrás (2012) para la elaboración de un guion educativo (storyboard):

- Planificar el proceso de enseñanza-aprendizaje redactando los objetivos y contenidos incluidos en la píldora.

- Llevar un control del nivel de conceptualización, de la cantidad de información que se maneja y del nivel del vocabulario expresado.

- Buscar un incremento progresivo de los estímulos sonoros y visuales para mantener la atención del espectador.

- Indicar las secuencias y las escenas para hacer una estimación del tiempo total del vídeo.

Estos guiones se escribieron inicialmente en inglés. Puesto que los videotutoriales debían grabarse además en los cuatro idiomas del proyecto (alemán, español, portugués y checo), se numeraron las frases de cada uno de los guiones y se pidió a los socios del proyecto que mantuviesen en sus traducciones esa numeración. Esto facilitaría el proceso de

\section{En función de los objetivos de aprendizaje establecidos se elaboraron cuatro videotutoriales: «Entorno y preparación», «Contenido y discurso», «Trucos valiosos» y «Aspectos técnicos»}


posproducción y de creación de materiales de apoyo, basados en texto, ya que los técnicos del departamento audiovisual siempre podían conocer la correspondencia en español de cada una de las frases.

\subsubsection{Desarrollo y producción}

La grabación de los vídeos se llevó a cabo en el estudio de grabación de la universidad, que se encuentra equipado con un sistema de producción y posproducción de vídeo integrado o TriCaster, un teleprompter o autocue, que permite a la persona leer el texto de su discurso al mismo tiempo que lo pronuncia, y un escenario con chroma key o «llave de color», que consiste en extraer un color de una imagen o vídeo (usualmente el verde o el azul) y reemplazar el área que ocupa ese color por otro fondo, con la ayuda de un equipo especializado o un ordenador.

En primer lugar, se dio a los guiones el formato adecuado para su inclusión en el autocue. Después, se grabó a los oradores en el escenario delante del chroma key. Y, finalmente, se llevó a cabo la posproducción mediante el software Adobe Premier Pro:

- Selección de secuencias grabadas, ordenándolas y acoplándolas según el orden previsto en el guion.

- Sustitución del fondo por un escenario digital con la inclusión de los materiales de apoyo.

- Incorporación de cabeceras y música de fondo.

- Montaje final en un solo clip de todas las escenas.

El formato de los videotutoriales fue un plano medio, con un orador en primer plano y un fondo en el que se iban reproduciendo los materiales de apoyo para hacer más comprensible su discurso. Dependiendo del contenido que se quiso ilustrar, se elaboraron tres tipos de materiales (Borrás, 2012):

- Screencast. Grabación de un vídeo que captura el contenido de la pantalla del ordenador. Este tipo de recurso es muy apropiado para enseñar a utilizar un software concreto o un programa informático, por lo que fue el elegido para ilustrar el videotutorial sobre el

Dependiendo del contenido que se quiso ilustrar, se elaboraron tres tipos de materiales: screencast, presentación y videoejemplos uso y el manejo de una herramienta de edición de vídeos (Oud, 2009). 
- Presentación. Sucesión de diapositivas con imágenes y textos que permitían una mejor asimilación de los conceptos más abstractos.

- Videoejemplos. Grabación de situaciones reales que ejemplifican acciones, actitudes o momentos descritos por el orador principal y cuya visualización clarifica el contenido expuesto.

\subsubsection{Integración, despliegue y testeo}

En esta fase, las tareas de preproducción, producción, desarrollo y testeo se desempeñan con naturalidad y flexibilidad en el propio entorno virtual videotutorial, de manera que el producto final se va construyendo y cerrando de forma lineal, guiado fundamentalmente por el diseño conceptual para tutoriales y vídeos de ejemplo asociados a los resultados $\mathrm{V}$ y VI, respectivamente.

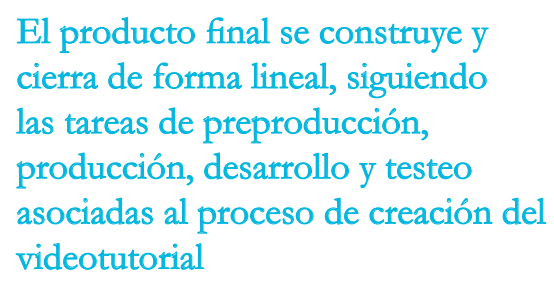
cierra de forma lineal, siguiendo las tareas de preproducción, producción, desarrollo y testeo videotutorial

\section{Nexos de evaluación del resultado entre proyectos}

Como se ha comentado con anterioridad, los videotutoriales audivisuales para la generación del curriculum vitae en vídeo constituyen el resultado núm. 2 del proyecto CVTube y se transfieren al resultado núm. 6 del proyecto RefuSkills (véase figura 2).

El proyecto CVTube incluye en su memoria técnica una evaluación previa de estos videotutoriales antes de llegar a la versión final de los mismos:

- 02/T6. Evaluación interna y finalización de los tutoriales online:

- Resultado IX. 1 informe de evaluación de cada socio (7 en total).

- Resultado X. Tutoriales mejorados en:

- Inglés.

- Alemán.

- Checo.

- Español.

- Portugués. 
Figura 2. Representación gráfica de la ubicación y de la generación del curriculum vitae videotutorial audiovisual para su posterior transferencia y reusabilidad entre proyectos

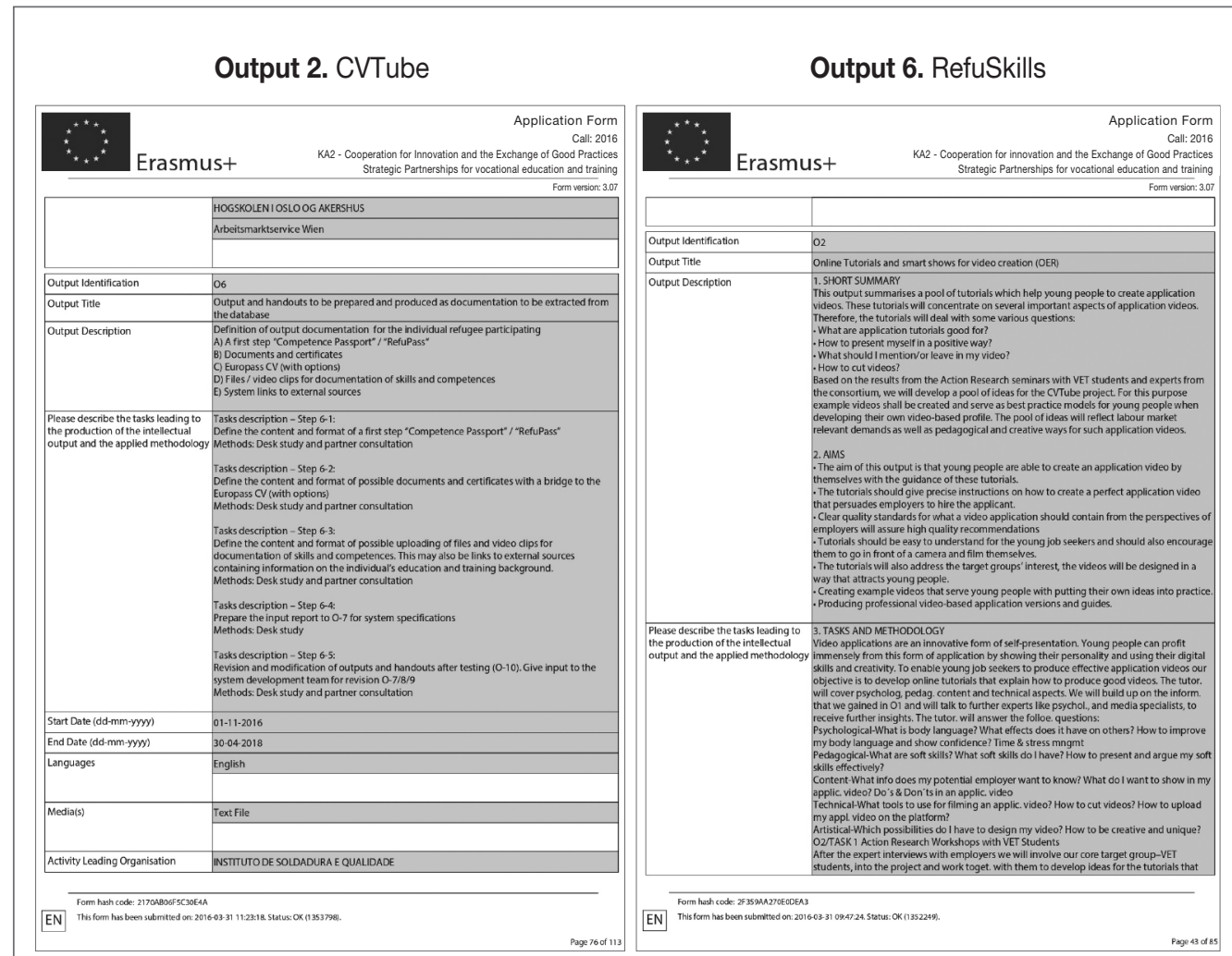

\section{Impacto de los resultados}

Cada uno de los proyectos incluye un plan de difusión de resultados generales específico. Para conocer el verdadero impacto de los resultados, se describen, a continuación, las características generales de dichos planes:

- CVTube. La difusión interna se centra en los contactos de las instituciones asociadas que no participan directamente en el proyecto. Se les informará regularmente sobre el progreso del proyecto. La difusión externa se enfocará en el nivel local-regional. La difusión en este nivel tendrá como objetivos: 
- Concienciar a los jóvenes sobre cómo evitar el desempleo y la exclusión social, además de conseguir posibles oportunidades laborales.

- Generar interés entre los jóvenes para participar en las diferentes fases de evaluación del proyecto.

- Involucrar a expertos que puedan beneficiarse de los resultados del proyecto.

- Garantizar la participación activa de todos ellos en los diferentes eventos de difusión.

Además, también es importante hacer difusión del proyecto entre los grupos objetivo del mismo a nivel europeo. La difusión se llevará a cabo por diferentes vías, tales como apariciones en medios tradicionales (artículos de prensa, anuncios de televisión/radio, etc.), medios digitales y redes sociales (Facebook, YouTube, Twitter, etc.).

- RefuSkills. En relación a la difusión del proyecto, el coordinador es el que hará siempre la presentación de los resultados a las organizaciones interesadas. En general, los socios del consorcio están igualmente obligados a informar sobre el proyecto a través de sus redes llevando a cabo eventos de difusión y conferencias. Se facilita a todos los socios una plantilla para realizar el seguimiento del plan de difusión local, y en ella se incluirán reuniones, eventos, redes sociales y cualquier actividad realizada con ese propósito.

\subsection{Difusión de resultados asociados al proyecto CVTube}

Planificada inicialmente para coincidir con las últimas fases de desarrollo, previas al lanzamiento del videotutorial audiovisual, actualmente estas acciones responden a los objetivos planteados en la fase III del proyecto CVTube, plataforma online para curriculum vitae en vídeo.

La plataforma CVTube incluirá tres secciones:

- Una sección al intercambio de curriculum vitae y curriculum vitae en vídeo entre jóvenes y posibles empleadores. Permite a los usuarios crear perfiles personales en los que incluir los curriculum vitae en formato audiovisual.

- Otra sección contiene los videotutoriales que deberían ayudar a los jóvenes a crear sus propios curriculum vitae en vídeo.

- Una tercera sección está dedicada a la colaboración, al intercambio de información y a la ayuda entre pares en la creación de sus perfiles y vídeos. La plataforma dispondrá de un área de chat para mensajes abiertos y privados que permitirá a los usuarios hacer preguntas y escribir comentarios. 
Una característica que es muy innovadora y complementa los tutoriales es la posibilidad de solicitar revisiones del propio perfil y, especialmente, los vídeos de otros usuarios (revisión por pares) y de un tutor especial (revisión experta) que se mantendrá más allá de la duración del proyecto.

En el caso de CV'Tube, el impacto inicial del proyecto se estima en un total de 410 personas

Además, la plataforma servirá como una bi-

blioteca digital abierta: los profesores podrán descargar el manual elaborado en la fase IV en formato pdf y en libro electrónico en todos los idiomas del proyecto, además del inglés.

La plataforma CVTube será accesible tanto para los jóvenes que buscan empleo como para los posibles empleadores. Los vídeos y perfiles creados tendrán diferentes niveles de visibilidad para una mejor protección y control de datos por parte del usuario. El usuario puede, por ejemplo, elegir si su perfil es accesible de forma abierta a través de un motor de búsqueda o si se convierte en anónimo con datos personales ocultos para los espectadores no autorizados.

En el caso de CVTube, el impacto inicial del proyecto se estima en un total de 410 personas:

- 20 personas involucradas en las entrevistas llevadas a cabo en la fase I. El perfil de estas personas es el de responsables de recursos humanos y empleadores que se beneficiarán al recibir información sobre una forma innovadora de solicitud de empleo.

- 40 estudiantes de formación profesional que participen en la fase II. Estos estudiantes se beneficiarán al recibir un certificado de participación y poder incluirlo en sus propios curriculum vitae.

- 20 estudiantes universitarios que participen en la fase II. Los estudiantes universitarios pueden conseguir ideas interesantes para desarrollar los tutoriales en línea interactivos y actualizados.

- 40 participantes en el testeo de la plataforma desarrollada en la fase III (jóvenes y responsables de recursos humanos y empleadores).

- 40 profesores de formación profesional y formadores que participen en el testeo del manual elaborado en la fase IV.

- 200 personas que asistirán a los eventos de difusión. Ellos se beneficiarán obteniendo información sobre los curriculum vitae en formato audiovisual y sobre la plataforma CVTube.

- 50 participantes a la conferencia final. Ellos también obtendrán información sobre los curriculum vitae en formato audiovisual y sobre la plataforma CVTube, además de tener la posibilidad de hacer networking y de encontrar oportunidades de colaboración con otros participantes. 


\subsection{Difusión de resultados asociados al proyecto RefuSkills}

Respecto al proyecto RefuSkills, teniendo en cuenta el número de refugiados que llegaron a los países de los socios del proyecto en 2015 y calculando la progresión de este flujo durante los años 2017 y 2018, se estima que el proyecto puede impactar en un total de 6.000 personas.

Además, el número de refugiados que hay en el campo de refugiados Nizip-2, en Gaziantep, ubicado en Turquía, en el que se llevó a cabo el testeo asociado al resultado 10, es de 50.000 individuos (usuarios potenciales de los videocurrículums).

\section{La transferibilidad de los resultados y el conocimiento}

En la comunicación «Mejorar la transferencia de conocimiento entre las instituciones de investigación y la industria en toda Europa» (Comisión Europea, 2007) se pone de manifiesto un punto de partida para las discusiones sobre un marco europeo común para la transferencia de conocimiento. Esta transferencia desempeña un papel notable en el Espacio Europeo de Investigación.

La «transferencia de conocimiento» consiste en la gama de actividades que apuntan a capturar y transmitir conocimiento (ya sea explícito, como en patentes, o tácito, como el know-how), habilidades y competencias de aquellos que los generan a aquellos que los transformarán en resultados económicos. Incluye tanto actividades comerciales como actividades no comerciales, tales como colaboraciones de investigación, consultoría, licencias, creación de spin-off, movilidad de investigadores y publicación. Por consiguiente, la transferencia de conocimiento es un concepto más amplio que la «transferencia de tecnología»: incluye otros canales de transferencia, como la movilidad del personal o las publicaciones.

La transferencia de conocimiento es beneficiosa para todas las categorías de partes interesadas, que incluye universidades, centros públicos de investigación e industria. La transferencia efectiva de conocimientos constituye un mecanismo clave del Espacio Europeo de Investigación y garantiza que la investigación financiada con fondos públicos tenga un impacto efectivo en la competitividad de la Unión Europea.

Por consiguiente, como demostramos en el presente trabajo, esta premisa se cumple, ya que la universidad generadora de los resultados se ha involucrado en la transferencia de dicho conocimiento, a pesar de que, en opinión de la Comisión, un tema tan fundamental pone de manifiesto que «muchas universidades europeas aún subestiman los beneficios potenciales de compartir conocimiento con la economía y la sociedad» (Comisión Europea, 2007). 
En este sentido, los beneficios de la transferencia de conocimiento, en otras palabras, la explotación de la investigación, van más allá del simple retorno financiero. El beneficio también radica en una serie de otros beneficios menos tangibles, como ayudar a las instituciones de investigación a centrar su estudio en las necesidades más amplias de la sociedad y la industria.

En la era de la información, el conocimiento y la tecnología juegan un papel crucial en el avance del crecimiento económico nacional. El papel de las universidades en la transferencia de conocimiento del sector académico al sector privado es fundamental.

Los avances en las tecnologías de la información y la comunicación (TIC) digitales también han facilitado un mayor intercambio de información científica, dentro y fuera de la esfera académica, que tiene implicaciones directas para las economías nacionales. En las últimas décadas, el papel de las universidades se ha extendido más allá de la «investigación tradicional y de la misión de la enseñanza» (Rossi y Rosli, 2013, p. 3). La contribución de las universidades a la producción, a la transmisión y a la difusión del conocimiento científico significa que estas instituciones también se perciben como agentes económicos ${ }^{2}$. La revolución de la información nos indica que los investigadores académicos tienen un mayor acceso a la información y poseen un conjunto más amplio de habilidades y experiencia; por lo tanto, los investigadores aplican los conocimientos adquiridos y las habilidades desarrolladas para investigar y generar nuevas ideas que pueden resultar positivas tanto en la mejora de la calidad y en la eficiencia de los productos y procesos como en el desarrollo de soluciones innovadoras para abordar desafíos sociales clave (Hoorens et al., 2013).

Así pues, la premisa de la transferibilidad de los resultados (véase figura 3) -los resultados potencialmente transferibles (videotutoriales para la generación de los videocurrículums, entendidos como conocimiento generado)- se cumple en este trabajo desde dos perspectivas diferenciadas:

- Transferencia directa de los resultados gratuitos y reusables a los beneficiarios (a la sociedad, a los estudiantes del ámbito de la formación profesional y de la formación superior, al mundo empresarial, a los stakeholders y a los refugiados que actualmente se encuentran en el campo de refugiados Nizip-2, en Turquía, entre otros).

- Transferencia de proyecto a proyecto. Reusabilidad del resultado, ampliando notablemente el número de potenciales usuarios finales.

2 <https://eur-lex.europa.eu/LexUriServ/LexUriServ.do?uri=COM:2003:0058:FIN:en:pdf>. 
Figura 3. Representación gráfica del potencial de transferibilidad de los resultados generados

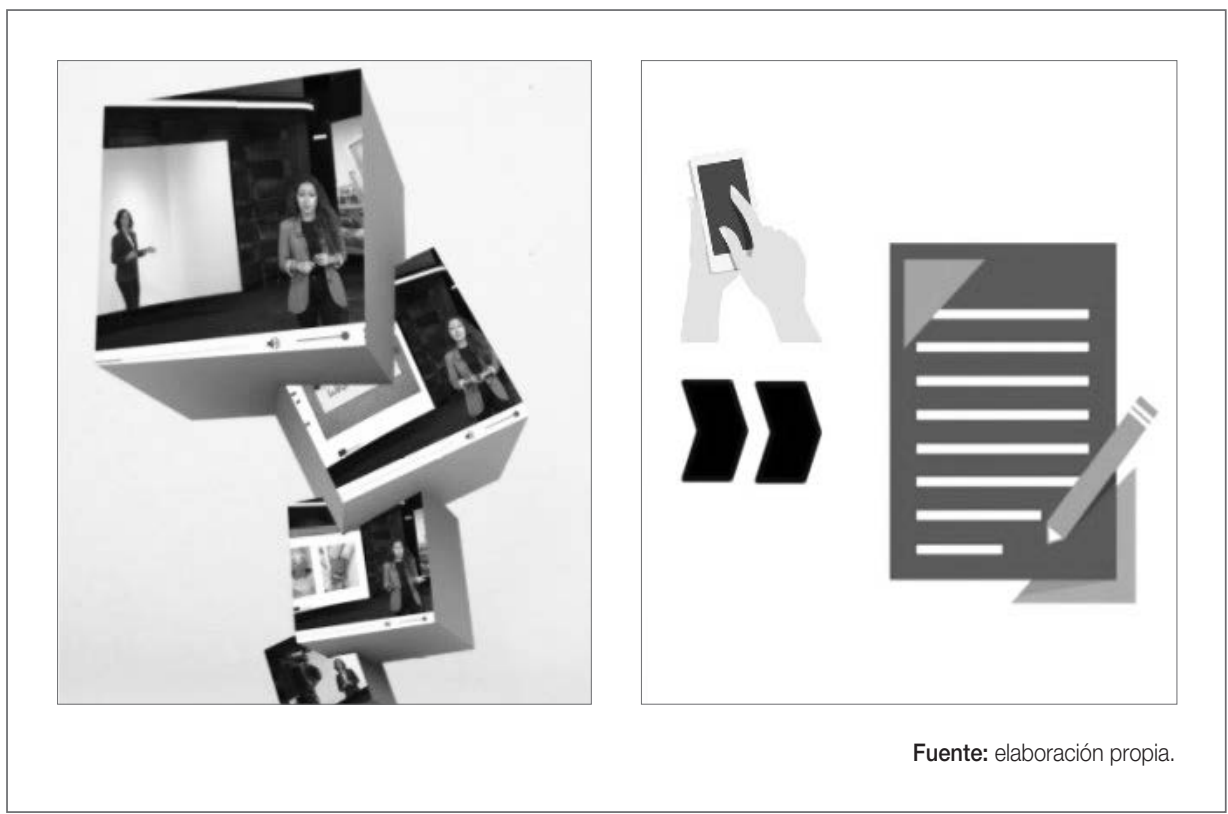

\section{Evaluación}

La evaluación de los resultados es doble. Por una parte, está la evaluación que hacen los propios usuarios de los videotutoriales y, por otra parte, las evaluaciones que se llevan a cabo dentro de cada uno de los proyectos y que abarcan tres aspectos diferenciados:

- Plataforma (contenedor).

- Usuario final (perceptor del resultado final).

- Contenido (videotutoriales-audiovisuales).

Cuadro 5. Evaluación asociada a la plataforma 03/T2-T3

\section{3/T2-T3. Pruebas, ajustes y finalización de la prueba piloto de la plataforma online}

02/T6. Evaluación interna y finalización de los tutoriales online.

- Resultado IX. 1 informe de evaluación de cada socio (7 en total). 
03/T2-T3. Pruebas, ajustes y finalización de la prueba piloto de la plataforma online

02/T2. Seminarios de investigación dirigidos a estudiantes:

- Resultado II. 2 seminarios dirigidos a estudiantes de formación profesional en checo y español.

- Resultado III. 2 seminarios dirigidos a estudiantes de formación superior en alemán y portugués.

010. ResfuSkills. Desarrollo de un testeo por pasos en los países socios para depurar errores, hacer modificaciones y mejorar la funcionalidad.

010/T1. Preparar los procedimientos de testeo en los países socios basándose en la información de los O1, 02, 04 y 09.

010/T2. Revisión de los procedimientos de testeo.

010/T3. Recopilación de los comentarios de retroalimentación.

010/T4. Dar la retroalimentación sobre las modificaciones propuestas y la depuración de errores al equipo de desarrollo.

Fuente: elaboración propia.

\section{Resultados}

Descripción técnica de los resultados generados: tutoriales en formato vídeo de corta duración en los que se alternan diferentes recursos audiovisuales (capturas de pantalla, vídeos en segundo plano, imágenes, etc.) con la imagen del orador que está explicando los contenidos.

Número total de vídeos generados: 20.

Número total de idiomas:

- Inglés.

- Portugués.

- Español.

- Alemán.

- Checo.

Disponibles en la sección «Innovación» (proyectos) de la web de la UDIMA: <https:// www.udima.es/es/innovacion/proyectos.html>. 
Figura 4. Ubicación de los videotutoriales en la web de la UDIMA

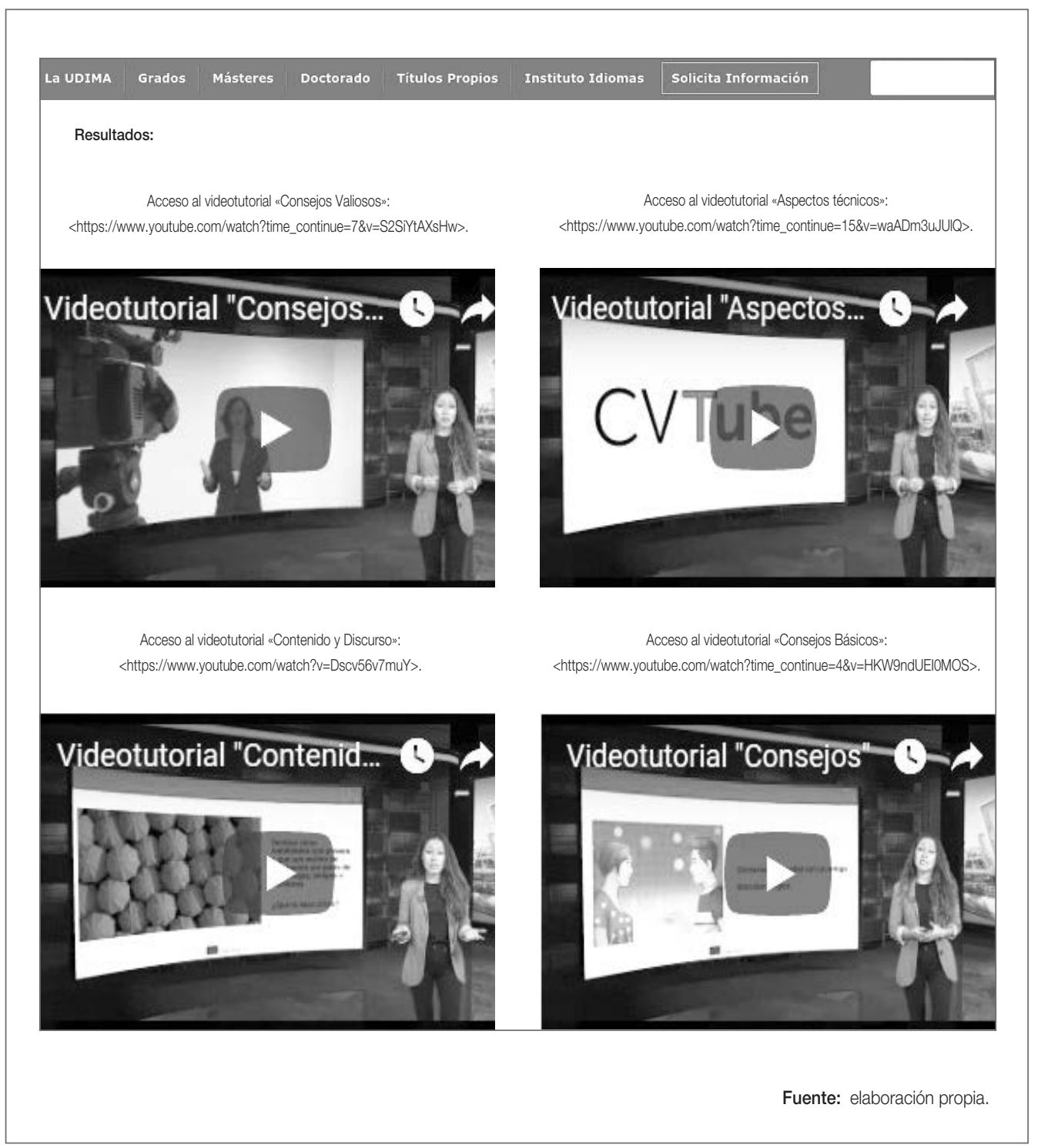

\section{Conclusiones}

El curriculum vitae se presenta como una excelente herramienta de branding para la búsqueda activa de empleo y/o el acceso al mercado laboral. 
Trabajar la realización de videocurrículums ha de abordarse desde dos perspectivas, como proceso y como resultado, siendo ambas importantes y complementarias. Lo importante de este tipo de actividad no es únicamente el producto final, que viene representado por la adquisición de competencias por parte de los alumnos para realizar una grabación en la que se fomente una adecuada imagen que ayude a la marca personal y a la reputación del mismo. No se trata solamente de entrenar al estudiante acerca de la mejor imagen que pueda ofrecer en la grabación, sino trabajar con él para que la imagen que muestre sea la mejor posible y fruto de un proceso de trabajo personal que lo predisponga positivamente hacia la inserción laboral efectiva (Climent-Rodríguez et al., 2012).

Los mismos autores coinciden al afirmar que «el videocurrículum-branding, como metodología docente desarrollada en el aula, permitirá que el docente ayude al alumno, como futuro demandante de empleo, en dirigir su atención en aquellas áreas más deficitarias en relación a su próxima inserción laboral, así como sus fortalezas y aquellas características personales que le añaden valor y marcan las diferencias.

Con todos estos datos se podrán llevar a cabo programas de entrenamiento de cara a realizar una grabación de su imagen con éxito. Dicho entrenamiento consistirá en modificar/mejorar la imagen de las personas ante un determinado empleo, dotándoles de estrategias y recursos personales adecuados para su inserción laboral» (p. 1.413).

El posicionamiento en favor de la potenciación del conocimiento abierto, mediante la aportación de contenidos y la gestión de vídeos gratuitos y online, propicia la transferibilidad en diferentes contextos empresariales y educativos. Este valor no puede desestimarse: la motivación, la flexibilidad, la modularidad y la reusabilidad propician un alto grado de aplicabilidad.

El modelo de producción de vídeo y los resultados expuestos coinciden con el planteamiento de vídeos en segmentos cortos de Hansch et al. (2015), separados por otros contenidos o actividades autoevaluativas. Se pone en discusión, no obstante, el valor añadido que ofrece el vídeo para el aprendizaje, considerando que, si bien la calidad del audio es esencial, las producciones profesionales y de alto nivel no siempre redundan en una mejora sustantiva del resultado. Aunque se coincide en que el diseño de producción debe ser consecuente con el contexto, la audiencia y la naturaleza del contenido de la finalidad, los resultados obtenidos muestran que la óptima calidad de todo el producto, la diversidad de formatos y de procesos redunda en resultados globales más eficaces, aprovechables, reutilizables y con una vigencia mayor.

Con los resultados generados, no solo se favorece el acceso al mercado laboral, sino que se ha podido cumplir la premisa de transferibilidad de conocimiento a la sociedad, en general, y al mundo empresarial, a la que todo proyecto de estas características debería aspirar. 


\section{Referencias bibliográficas}

Best, S. (2015). Education in the interregnum: an evaluation of Zygmunt Bauman's liquidturn writing on education. British Journal of Sociology of Education, 1-18.

Borrás Gené, O. (2012). Píldoras formativas y videojuegos aplicados al estudio de la ingeniería acústica (Tesis doctoral). Escuela Universitaria de Ingeniería Técnica de Telecomunicación, Universidad Politécnica de Madrid.

Clark, J. M. y Paivio, A. (1991). Dual coding theory and education. Educational Psychology Review, 3(3), 149-70.

Climent-Rodríguez, J. A., Navarro-Abal, Y. y Ortega-Campos, E. (2012). El videocurrículum-branding como recurso digital para el desarrollo de competencias de búsqueda de empleo en los futuros egresados. En D. C. Sanchiz, A. Jaén Martínez, E. López Meneses, A. H. Martín Padilla y L. Molina García (Dirs.), I Congreso Virtual Internacional sobre Innovación Pedagógica y Praxis Educativa INNOVAGOGÍA 2012.

Contreras-Espinosa, R., Eguia-Gómez, J. y Solano Albajes, L. (2016). Investigaciónacción como metodología para el diseño de un serious game. RIED. Revista Iberoamericana de Educación a Distancia, 19(2). Recuperado de <http://revistas.uned.es/ index.php/ried/article/view/15624/14276> (consultado el 5 de enero de 2018).

Elliott, J. (2005). El cambio educativo desde la investigación-acción. Madrid: Morata.

European Commission. (4 April 2007). Knowled ge Transfer Between Research Institutions and Industry-Frequently Asked Questions. MEMO/07/127. Bruselas. Recuperado de $<$ http://europa.eu/rapid/press-release_MEMO -07-127_en.htm> (consultado el 2 de febrero de 2018).
Gértrudix Barrio, M., Rajas Fernández, M. y Esteban, N. (2015). URJC online. Plan estratégico para la mejora de la enseñanza en entornos virtuales. En L. Bengochea Martínez, C. D. Varela Báez y A. Miñán Espigares (Coords.), Formación virtual inclusiva y de calidad para el siglo XXI: actas del VI Congreso Internacional sobre Calidad y Accesibilidad de la Formación Virtual (CAFVIR) (pp. 228-234). Granada, España: Universidad de Granada.

Gértrudix Barrio, M., Rajas Fernández, M. y Álvarez García, S. (2017). Metodología de producción para el desarrollo de contenidos audiovisuales y multimedia para MOOC. RIED. Revista Iberoamericana de Educación a Distancia, 20(1), 183-203.

Gértrudix, M., Álvarez, S., Galisteo, A., Gálvez, M. ${ }^{a}$ C. y Gértrudix, F. (2007). Acciones de diseño y desarrollo de objetos educativos digitales: programas institucionales. RUSC. Universities and Knowledge Society Journal, 4(1), 14-25.

Given, L. (2008). The SAGE Encyclopedia of Qualitative Research Methods. SAGE Publications.

González-Sancho, C. y Vincent-Lancrin, S. (2016). Transforming education by using a new generation of information systems. Policy Futures in Education. doi: 10.1177/1478210316649287.

Guerrero Rodríguez, J. H. y Vela González, P. A. (2013). Investigación participativa (IAP). Metodologías, Estrategias y Herramientas Didácticas para el Diseño de Cursos en Ambientes Virtuales de Aprendizaje en la Universidad Nacional Abierta y a Distancia (pp. 10-14). UNAD.

Guo, P. J., Kim, J. y Rubin, R. (2014). How video production affects student engagement: an empirical study of MOOC videos. 
Proceedings of the First ACM Conference on Learning@ Scale Conference (pp. 41-50). Atlanta, Georgia, EE. UU.

Hansch, A., Hillers, L., McConachie, K., Newman, C., Schildhauer, T., y Schmidt, P. (2015). Video and Online Learning: Critical Reflections and Findings from the Field.

Hoorens, S., Ghez, J., Guerin, B., Schweppenstedde, D., Hellgren, T., Horvath, V. ... y Kobzar, S.(2013). Europe's Societal Challenges: An Analysis of Global Societal Trends to 2030 and their Impact on the EU. Rand Europe. Recuperado de <https:// www.rand.org/content/dam/rand/pubs/ research_reports/RR400/RR479/RAND_ RR479.pdf> (consultado el 5 de enero de 2018).

Iding, M. (2000). Can strategies facilitate learning from illustrated science texts? International Journal of Instructional Media, 27(3), 289-307.

López Meneses, E., Vázquez Cano, E. y Ramón Graván, P. (2015). Análisis e implicaciones del impacto del movimiento MOOC en la comunidad científica: JCR y Scopus (2010-13). Comunicar, 22(44), 73-80. Recuperado de <https://www.revistaco municar.com/index.php?contenido=deta lles\&numero $=44 \&$ articulo $=44-2015-08>$ (consultado el 3 de marzo de 2018).

Mayer, R. y Moreno, R. (2007). A cognitive theory of multimedia learning: implications for design principles. En F. T. Durso (Ed.), Handbook of Applied Cognition (2. ${ }^{\mathrm{a}}$ ed.). Wiley. ISBN: 978-0-470-01534-6.

Mayer, R. E., Steinhoff, K., Bower, K. y Mars, R. (1995). Agenerative theory of textbook design: using annotated illustration to foster meaningful learning of science text.
Educational Technology Research and Development, 43(1), 31-44.

Oud, J. (2009). Guidelines for effective online instruction using multimedia screencasts. Reference Services Review, 37(2), 164-177.

Paivio, A. (1986). Mental Representations: A Dual Coding Approach. New York: Oxford University Press.

Paulson, L. F., Paulson, P. R. y Meyer, C. (1991). What makes a portfolio a portfolio? Educational Leadership, 48(5), 60-63.

Picarra, M. (2015). Open Access to Scientific Information: Facilitating Knowledge Transfer and Technological Innovation from the Academic to the Private Sector. Recuperado de <http://www.pasteur 4oa.eu/sites/pasteur4oa/files/resource/ Brief_OA\%20and\%20knowledge\%20 transfer\%20to\%20the \%20private\%20 sector.pdf> (consultado el 4 de marzo de 2018).

Rossi, F. y Rosli, A. (19 July 2013). Indicators of university-industry knowledge transfer performance and their implications for universities: evidence from the UK's HE-BCl Survey. CIMR Research Working Paper Series, 13, 3. Recuperado de $<$ https://www.researchgate.net/publica tion/294583007_Indicators_of_universityindustry_knowledge_transfer_performance_ and_their_implications_for_universities_evi dence_from_the_UK's_HE-BCI_survey> (consultado el 16 de marzo de 2018).

Sweller, J. y Chandler, P. (1994). Why some material is difficult to learn. Cognition and Instruction, 12(3), 185-233.

Treichler, D. G. (1967). Are you missing the boat in training aid? Film and A-V Communication, 1, 14-16. 


\section{Ũ Grados oficiales}

\section{Magisterio de Educación Infantil}

Hoy en día los centros educativos tienen una imperiosa necesidad de disponer de personal especializado, capaz de hacer frente a las necesidades educativas de la etapa infantil, de acuerdo con los conocimientos ya logrados por las diversas ciencias que hoy se ocupan de los niños en los primeros años de su vida, así como de los logros relativos al desarrollo de la inteligencia, la emocionalidad y la formación de la personalidad temprana, resultantes de estudios recientes sobre el desenvolvimiento de la mente infantil.

\section{Magisterio de Educación Primaria}

Son objetivos de la educación primaria, entre otros: conocer y apreciar los valores y las normas de convivencia, aprender a obrar de acuerdo con ellas, prepararse para el ejercicio activo de la ciudadanía y respetar los derechos humanos, así como el pluralismo propio de una sociedad democrática. También, desarrollar hábitos de trabajo individual y de equipo, de esfuerzo y responsabilidad en el estudio, así como actividades de confianza en uno mismo, sentido crítico, iniciativa personal, curiosidad, interés y creatividad en el aprendizaje.

\section{Menciones en los grados de Magisterio de Educación Infantil y de Magisterio de Educación Primaria}

Mención en Lengua Inglesa

Mención en Pedagogía Terapéutica

Mención en Audición y Lenguaje

Mención en Tecnología Educativa

Mención en Enseñanza de la Religión Católica

\section{Curso de adaptación al grado}

Este curso de adaptación al grado ofrece a los maestros diplomados en la Especialidad de Educación Infantil o Primaria la posibilidad de obtener formación en campos determinados dentro del ejercicio profesional docente en estas etapas, a través de las menciones cualificadoras mencionadas anteriormente.

El objetivo principal del plan de estudios de este curso de adaptación al grado es contribuir a la actualización de la formación de los maestros diplomados. La aplicación de las TIC a la educación y de líneas pedagógicas innovadoras, fruto de la investigación en educación, hacen necesaria la actualización de los conocimientos didácticos de los diplomados y la formación de los maestros en investigación e innovación.

Al finalizar el curso de adaptación se obtiene el título de grado en Magisterio de Educación Infantil o en Magisterio de Educación Primaria.

La docencia en la etapa de educación infantil o primaria es una profesión regulada. Los graduados en Magisterio de Educación Infantil o en Magisterio de Educación Primaria tienen como principal salida profesional el trabajo como profesores en estas etapas, tanto en centros públicos como concertados y privados.

Si bien otras salidas profesionales para estos títulos pueden ser:

- Participación en proyectos educativos de organismos e instituciones (centros culturales, museos, asociaciones, ONG, etc.).

- Centros de educación para adultos.

- Centros de ocio y tiempo libre.

- Participación en programas de extensión educativa (actividades extraescolares, actividades de apoyo, etc.).

- Diseño y elaboración de materiales didácticos.

- Participación en proyectos de atención a la infancia y familiar. 